\title{
Riqueza y distribución de la flora urbana de Santiago de Chile: una aproximación basada en interpolación IDW
}

\author{
Riqueza e distribuição da flora urbana de Santiago de Chile: uma aproximação baseada em \\ interpolação IDW
}

\author{
Sergio Antonio Castro \\ Nicole Guerreo-Leiva \\ Maximiliano Bolados
}

Universidad de Santiago de Chile - Usach - Facultad de Química y Biología - Santiago - Chile

\section{Javier Alejandro Figueroa}

Universidad Central de Chile, Facultad de Arquitectura - Urbanismo y Paisaje - Santiago - Chile

\section{Resumen}

Desde el punto de vista ecológico, recién comenzamos a comprender los procesos y mecanismos que determinan diversidad florística en ciudades. En el presente artículo describiremos la riqueza y distribución espacial de especies de plantas al interior de la ciudad de Santiago, la metrópolis chilena. Para ello, se analizan los resultados obtenidos por un esfuerzo de muestreo florístico que busca reconocer la composición de diversidad vegetal presente en calles, plazas y sitios eriazos. Nuestros resultados muestran que la flora de Santiago muestra una amplia representación de plantas exóticas (85\% de las especies), en tanto que las nativas tienen una menor representación (15\% de las especies); ambos conjuntos de especies también difieren en la extensión distribucional que ocupan al interior de la ciudad ya que las especies exóticas se encuentran más ampliamente distribuidas que las nativas. Este patrón es concordante con lo que se ha documentado para otras ciudades del centro y sur de Chile; sin embargo, discrepan de aquellos obtenidos en ciudades del hemisferio norte (Europa y Estados Unidos), donde las especies nativas están mejor representadas. Llamamos la atención acerca de implementar medidas que propendan a incrementar la representación de especies nativas al interior de Santiago.

\section{Resumo}

Desde o ponto de vista ecológico, recém iniciamos a compreender os processos e mecanismos que determinam diversidade florística em cidades. No presente artigo, descrevemos a riqueza e distribuição espacial de espécies de plantas no interior da cidade de Santiago, a metrópole chilena. Para isto, analisam-se os resultados obtidos para um esforço de amostragem florístico que busca reconhecer a composição da diversidade vegetal presente em ruas, praças e sítios baldios. Os resultados indicam que a flora de Santiago mostra uma ampla representação de plantas exóticas (85 $\%$ das espécies), enquanto que as nativas uma menor representação (15\% das espécies); ambos conjuntos de espécies também diferem na extensão distribucional que ocupam no interior da cidade, uma vez que as espécies exóticas se encontram más amplamente distribuídas do que as nativas. Este padrão coincide com resultados documentados para outras cidades do centro e sul do Chile; entretanto, discrepam daqueles obtidos em cidades do hemisfério norte (Europa e Estados Unidos), onde as espécies nativas estão melhor representadas. Chama-se a atenção para a implementação de medidas que objetivem incrementar a representação de espécies nativas no interior de Santiago.

\section{Palabras clave}

Flora urbana. Plantas nativas. Plantas exóticas. Cartografía urbana. Santiago. Chile.

\section{Palavras-chave}

Flora urbana. Plantas nativas. Plantas exóticas. Cartografia urbana. Santiago. Chile. 


\section{Introducción}

El emergente desarrollo de la Ecología Urbana se ha centrado en comprender los procesos ecológicos y ambientales que emergen en respuesta a la creciente tendencia de urbanización (MCDONNELL e HASS, 2009; MCDONALD 2008). Bajo esta perspectiva, se estima que muchos de los cambios experimentados en zonas urbanas preludian las alteraciones observadas en ecosistemas naturales y viceversa (GRIMM et al., 2008). Por esta razón, las ciudades se han convertido en sistemas de estudio en ecología, que permitirían mejorar nuestra comprensión del cambio global y del modo en que los componentes biológicos y culturales se interrelacionan en un escenario espacialmente concreto (REDMAN et al., 2004; COLLINS et al., 2000). Se considera que los avances en esta área permitirán establecer las bases ecológicas y ambientales para mejorar el bienestar humano (ECKERT e KOHLER, 2014), entendiendo además que las urbes constituyen unidades geopolíticas susceptibles de planificar e intervenir (FELSON et al., 2013).

Recientes estimaciones señalan que la urbanización está aumentando de manera sostenida en las distintas regiones del globo. La información disponible muestra que sobre el $50 \%$ de los habitantes del planeta habita en ciudades (UN, 2011), aunque ellas no ocupan más del $3 \%$ de la superficie terrestre (ELLIS et al., 2010, SCHNEIDER et al., 2010). En Europa, América Latina y Estados Unidos, la población actual que reside en ciudades alcanza el 72, 78 y $80 \%$, respectivamente (UN, 2007; BROWN et al., 2005), pero de acuerdo a estimaciones de las Naciones Unidas, estos valores deberían aumentar en un $10 \%$ como promedio dentro de los próximos 40 años (UN, 2007). En el caso particular de Sudamérica, el porcentaje de la población que reside en ciudades varía entre los diferentes países, registrándose valores que oscilan entre $75 \%$ en Colombia y el $89 \%$ en Argentina (MAcGREGOR-FORS e ORTEGAALVAREZ, 2013). En este contexto, Chile es uno de los países con mayor población urbana en la región con una población urbana de 87 \% (UN, 2007), superando además, el promedio observado en varios países europeos.

Las ciudades conglomeran a las personas porque facilitan el acceso a recursos y servicios (MAcGREGOR-FORS e ORTEGA-ALVAREZ, 2013; KABISCH e HAASE, 2011; PICKETT et al., 2001). Sin embargo, la sustentación urbana no solo proviene de los procesos originados en su interior sino que también de los suplementos externos que se generan desde regiones o ecosistemas adyacentes (PICKETT et al., 2001). Además, según los mismos autores, esta situación ha causado profundas modificaciones en los patrones de uso del suelo, cambios que se traducen en la conversión de hábitats naturales en tierras de cultivo y que luego, fruto de la expansión urbana, pueden ser absorbidos por la ciudad como suelos urbanizados.

La relación entre urbanización y biodiversidad es estrecha (McDONALD et al., 2013; MCKINNEY, 2008). Tradicionalmente las ciudades han sido fundadas en regiones particularmente biodiversas, con el objeto de asegurar la provisión de recursos para el sustento humano (ARAUJO, 2003; CINCOTTA et al., 2000). Sin embargo, el crecimiento de las ciudades ha traído como consecuencia un progresivo impacto en su entorno, la fragmentación del paisaje y modificación del hábitat original para numerosas especies (LOSOSOVÁ et al., 2011; WERNER, 2011; TURNER et al., 2004). Desde el punto de vista ecológico, recién comenzamos a comprender los procesos y mecanismos implicados en la relación urbebiodiversidad (McDONNELL et al., 2009). 
Así, por ejemplo, la composición y distribución de especies de plantas al interior de las ciudades es el resultado de la acción de un conjunto de procesos de distinta naturaleza y de diversa escala (MARCO et al., 2010). Por una parte, a través de una serie de decisiones, los seres humanos determinamos qué y cuántas especies deseamos disponer en nuestro entorno; y por otra, las condiciones ambientales y de hábitat pueden afectar a esta diversidad, facilitando o impidiendo la sobrevivencia de estas especies (REYES-PAESKE et al., 2011). Como resultado, la flora urbana resultará conformada por un conjunto de especies de distintos orígenes evolutivos y geográficos, incluyendo especies nativas remanentes de la vegetación original y aquellas exó (Ilamadas también exóticas o alóctonas) desde otras regiones del planeta (KNAP et al., 2008; KOWARIK, 2011). El balance entre la composición de especies nativas y exóticas puede ser un primer paso para comprender las consecuencias que la urbanización tiene para la biodiversidad contenida, así como un primer indicador del efecto de la urbanización sobre la biodiversidad.

Hasta ahora, una baja proporción de las ciudades del mundo han sido estudiadas en términos de la composición de especies de plantas. La mayor parte de estos estudios han sido efectuados en ciudades europeas, en países que han gozado de una larga tradición botánica y paisajista (e. g., CELESTI-GRAPOW et al., 2013; KOWARIK et al., 2011, 2013; LA SORTE e PYSEK, 2009; GASTON et al., 2005; KÜHN et al., 2004). Interesantemente, en estas ciudades la representación de especies nativas suele superar a la representación de especies exóticas tanto a nivel de ciudades completas (LOSOSOVÁ et al., 2012a) como en los diferentes hábitat al interior de ellas: plazas, parques o calles (LOSOSOVÁ et al., 2012b). Estos autores, por ejemplo, analizando la composición y distribución florística en 32 ciudades de Europa central encontraron que la composición de plantas nativas fue de $48 \%$, de la exóticas arqueofitas (especies de plantas introducidas antes del año 1500) de $16 \%$, y de exóticas neofitas (especies de plantas introducidas después del año 1500) de $33 \%$. Reportaron, también, que en distintos tipos de hábitat urbanos, la diversidad de especies nativas siempre superó a las arqueofitas y neófitas (para definiciones ver LA SORTE et al., 2007). Por otra parte, sobre la base de información florística obtenida para 85 ciudades europeas y 25 no-europeas, Aronson et al. (2014) y La Sorte et al. (2014) documentaron que la representación de especies nativas registró una mediana de $52 \%$ en las 110 ciudades, y que la representación de especies nativas fue mayor en ciudades no-europeas (cercana al $70 \%$ ).

El estado del conocimiento en países europeos contrasta con lo que conocemos en otros continentes, incluyendo Chile. Por ejemplo, un primer esfuerzo por documentar la composición de la flora urbana a escala nacional fue realizado por Hoffman (1988), en su libro El Árbol Urbano en Chile. Este texto incluyó 105 especies de árboles urbanos para ciudades de Chile, de los que el $7 \%$ fueron nativos y el $93 \%$ exóticos. Más recientemente, Rodríguez et al. (2005) reconocieron 283 especies de árboles y arbustos para Chile, de los cuales el $43 \%$ fueron especies nativas y $57 \%$ exóticas; no obstante, estos autores indicaron que las ciudades de Chile presentan una mayor proporción de especies exóticas que de nativas, aunque esta diferencia o magnitud no ha sido cuantificada.

En este contexto, en el presente artículo describiremos el resultado de nuestro trabajo de muestreo florístico realizado al interior de la ciudad de Santiago. Esta iniciativa se estableció con el objeto de conocer la composición de diversidad vegetal presente en el espacio público de Santiago (calles, plazas y sitios eriazos), en el intento de conocer la representación de especies nativas y exóticas. Aunque algunos resultados ya han sido 
publicados previamente (FIGUEROA et al., 2014) o se encuentran en fase de preparación, en el presente capítulo extenderemos y complementaremos este análisis buscando describir la distribución espacial de la flora nativa y exótica presente en la ciudad de Santiago. Para ello, utilizaremos herramientas cartográficas que permitirán interpolar y visualizar en mapas los patrones de distribución de la diversidad florística.

\section{Material y métodos}

\section{Santiago de Chile}

Desde el punto de vista del ordenamiento territorial, en Chile una ciudad se define como un conglomerado humano que supera los 5.000 habitantes; los conglomerados de menor tamaño corresponden a categorías de pueblos, aldeas o caseríos (INE, 2005). La distribución de los conglomerados urbanos en nuestro país muestra una acentuada centralización, siendo la ciudad de Santiago aquella más poblada y de mayor área del país (Figura 1). El impacto que ha tenido el crecimiento de la ciudad de Santiago para alcanzar las dimensiones actuales, y qué capacidad de sustentación tiene este tipo de crecimiento para un país como Chile, constituye una inquietante pregunta que deberán resolver las futuras investigaciones. Así, Santiago (33을 $27^{\prime} 00^{\prime \prime} \mathrm{S}$; 700 40'00" O; Figura 2), progresivamente se ha transformado en la metrópolis del país, centro económico y político del Chile. Su extensión actual abarca unos $830 \mathrm{~km}^{2}$ (INE, 2005), aunque este valor varía dependiendo de lo que es considerado como borde urbano.

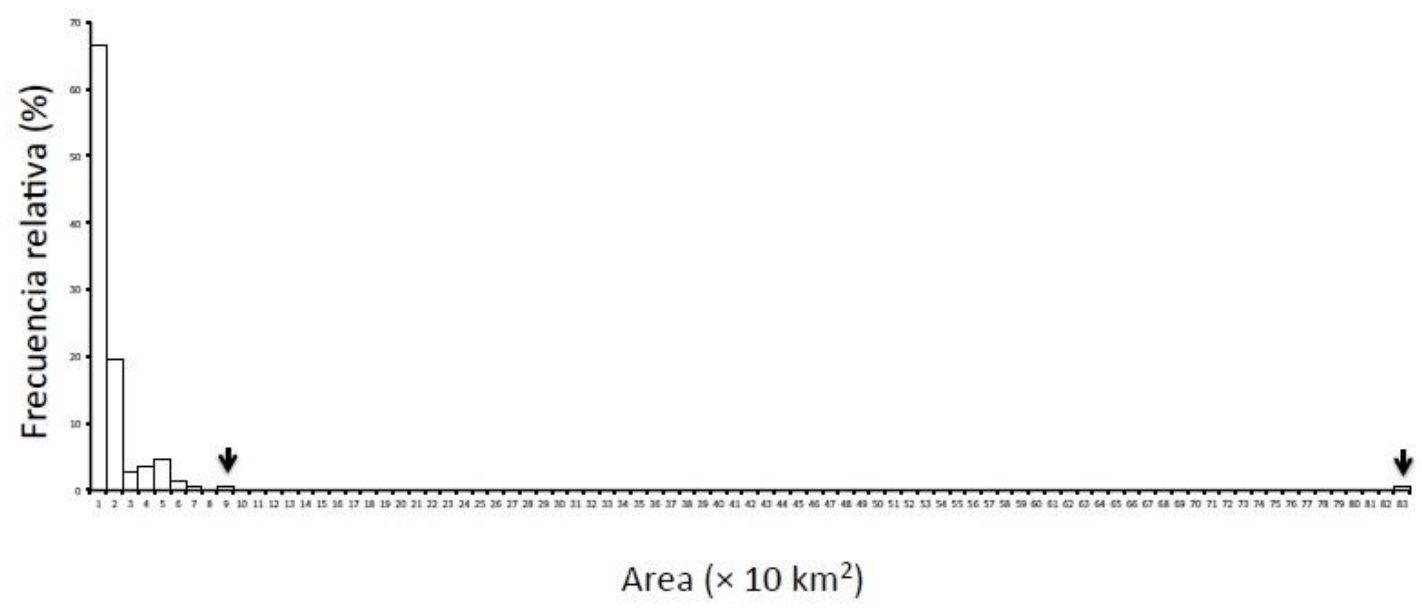

Figura 1. Distribución de frecuencia relativa (\%) de tamaños de ciudades en Chile, estimados a partir de su área $\left(10 \times \mathrm{km}^{2} ; \mathrm{n}=194\right.$ ciudades). Las dos flechas indican la ubicación de Santiago $\left(830 \mathrm{~km}^{2}\right)$ y de Viña del Mar $\left(90 \mathrm{~km}^{2}\right)$ a lo largo del gradiente, las dos ciudades más grandes del país (INE 2005). Nótese el hiato que separa a la ciudad de Santiago del resto de las urbes chilenas.

\section{Muestreo de la flora}

A partir del año 2013, iniciamos un muestreo de la flora santiaguina. Este muestreo implicó la visita de 230 sitios al interior de la ciudad seleccionados al azar mediante metodología SIG (Figura 2). Para estos efectos, en base a una fotografía aérea de Santiago se estableció un polígono del casco urbano. Luego, se usó el comando ArtcToolbox de ArcGis para 
distribuir aleatoriamente los puntos de muestreo al interior del polígono establecido. Tras visitar cada sitio, muestras de las plantas presentes fueron obtenidas en un transecto de $100 \mathrm{~m}$ sobre el espacio público, para su determinación en laboratorio, en tanto que otras especies fueron determinadas in situ. Las visitas fueron realizadas en primavera y verano, que son las estaciones de crecimiento para la mayoría de las plantas con flores. Una vez determinadas, las especies fueron registradas en una base de datos, indicando posteriormente su origen como nativo o exótico, así como su origen biogeográfico. Esta información fue establecida por consulta a diversas fuentes de publicaciones y bases de datos de referencia (FIGUEROA et al., 2014; RAY et al., 2014; CASTRO et al., 2005; ARROYO et al., 2000; MATTHEI, 1995; MARTICORENA e QUEZADA, 1985, 1987).

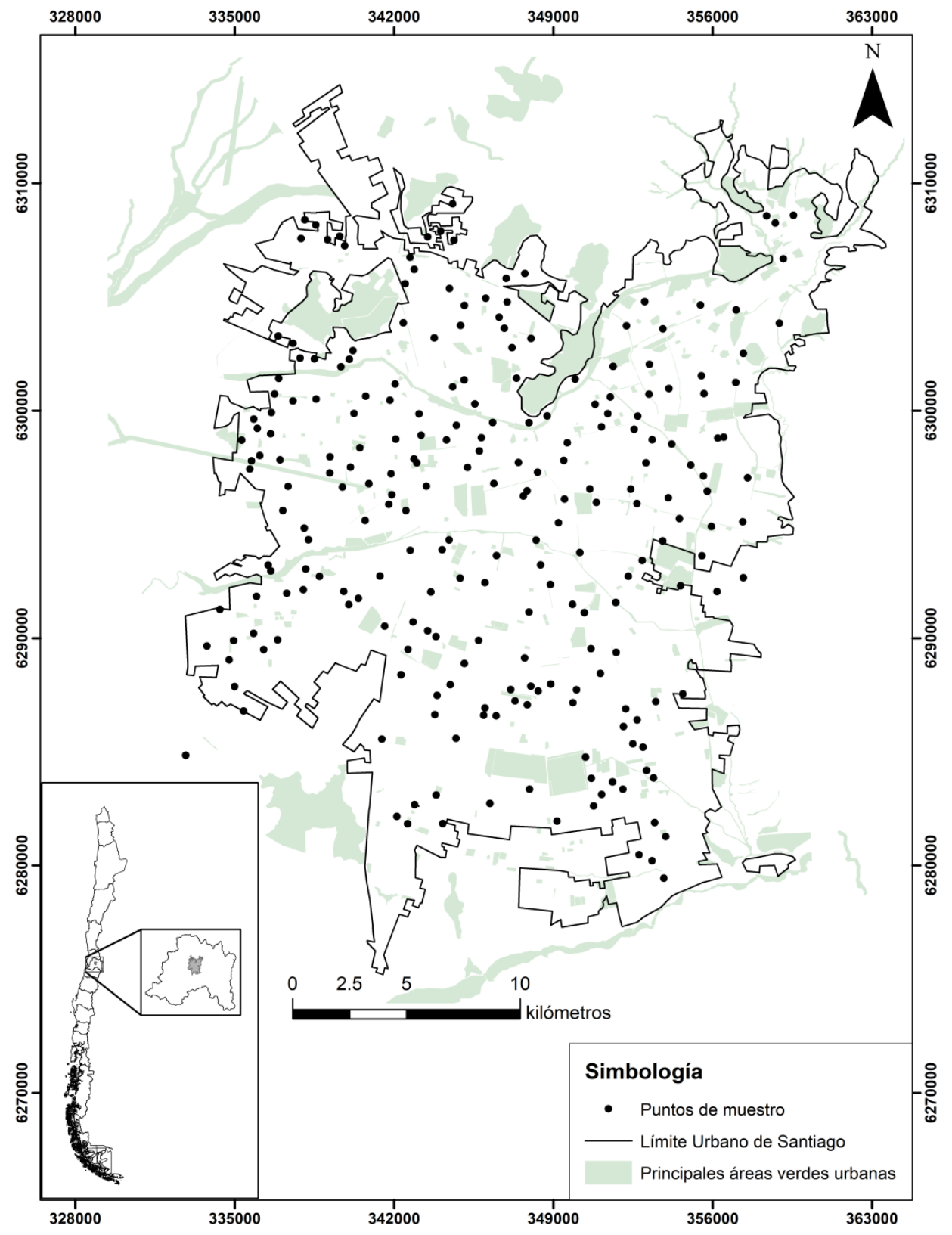

Figura 2. Distribución espacial de los puntos de muestreo de flora urbana $(n=230)$ implementados al interior de la ciudad de Santiago, Chile. 


\section{Densidad de especies y cartografía}

Con el objeto de establecer los patrones de diversidad y distribución de la diversidad de especies de plantas al interior de Santiago, se calculó la densidad de especies establecida como el número total de especies presentes en cada sitio muestreado dividido por el área total muestreada. Estos valores fueron expresados en unidades de número de especies por $\mathrm{m}^{2}$. Para estos efectos, para cada sitio se totalizó el número total de especies presentes y el área total muestreada, sin distinguir entre los distintos tipo de hábitat muestreados.

Los valores de densidad de especies fueron asignados a cada punto de muestreo, interpolando los valores a través del método de Distancia Inversa Ponderada (Inverse Distance Weighting, IDW, WATSON e PHILIP, 1985). El IDW es un método de interpolación espacial determinista, que supone que los puntos más próximos a la muestra son más parecidos entre si, de tal forma que existe una relación lineal de similitud entre un punto de valor conocido y sus vecinos. Esa relación de parentesco queda expresada por una función ponderada con la distancia. El valor que tomará un punto desconocido, pero en la vecindad del punto muestreado será la media ponderada por la distancia (Ecuación 1).

$$
Z(x, y)=\frac{\sum_{i, j} z(i, j) W(i, j)}{\sum_{i, j} W(i, j)}
$$

\section{Ecuación 1}

donde $Z(x, y)$ es el valor interpolado de un punto no muestreal, $z(i, j)$ es el valor de un punto muestreado al interior de un radio de búsqueda definido, y $W(i, j)$ son los pesos estadísticos asignados por el inverso de la distancia (Ecuación 2).

$$
W(i, j)=\frac{1}{D(i, j)^{n}} \quad \text { Ecuación 2 }
$$

donde $\mathrm{D}(i, j)$ corresponde a la distancia euclidiana desde el punto a considerar y un punto de valor conocido al interior de la vecindad definida, y $n$ es un exponente. Para efectos de la interpolación realizada en el software de información geográfica ArcGIS 10, el valor de la exponente $n$ fue 2, y la vecindad de puntos que influyen en la interpolación fue de 12 .

El procedimiento de interpolación fue implementado para el análisis total de la función de densidad de especies, así como para especies nativas y exóticas por separado.

\section{Resultados y discusiones}

La composición florística de la ciudad de Santiago obtenida a través de nuestro muestro estuvo conformada por 661 especies y otras 54 taxa no determinadas a nivel específico. Del total de especies identificadas, 568 fueron exóticas y 93 nativas, con una representación aproximada de 85 y $15 \%$, respectivamente. Esta proporción no difiere de la documentada previamente por Figueroa et al. (2014) $\left(\chi^{2}=0,45 ; g\right.$. I.= $\left.1 ; p>0,05\right)$, confirmando que en Santiago 
la composición de especies tiene una mayor representación de especies exóticas que de nativas.

La Figura 3 muestra la distribución espacial de la densidad de especies interpolada al interior de Santiago. La ciudad exhibe diversos focos (área en rojo) donde la diversidad es alta en términos relativos (cercano a una especie $/ \mathrm{m}^{2}$ ). Estos focos se encuentran distribuidos en todos los sectores de la ciudad, aunque preferentemente se localizan en sectores periféricos de la ciudad (Figura 3). Particularmente importante es destacar la extensión de cuatro áreas que destacan por su extensión ya que estas sitios están ubicados en las comunas de Renca e Independencia, La Florida, La Granja y San Bernardo (Figura 3); además, y en menor medida, se pueden reconocer otras áreas de menor extensión en otras comunas de los sectores oriente y poniente de la ciudad. En global, estos patrones de distribución de la diversidad de especies no se ajustan a los documentados por otros autores (HERNÁNDEZ e VILLASEÑOR, 2017; ESCOBEDO et al., 2006; DE LA MAZA, 2002). En efecto, estos autores han encontrado que la diversidad de especies de árboles y arbustos al interior de Santiago se correlaciona positivamente con el nivel socioeconómico de los barrios, por lo que la mayor diversidad se encuentra en el sector oriente de Santiago, en asociación con la ubicación de los grupos sociales de mayores recursos económicos (HERNÁNDEZ e VILLASEÑOR, 2017). Una posible explicación a este hecho es que nuestro estudio incluyó un mayor número de formas de vida (i. e. hierbas y arbustos), en tanto que los estudios previos solo han considerado árboles.

Santiago fue fundado en 1541 por colonos españoles, y permaneció sin experimentar gran desarrollo ni crecimiento tras 300 años de régimen colonial. Con la instauración de la República a inicios del siglo XIX, Santiago comenzó a crecer, hecho que suscitó interés por la ornamentación del espacio público. Diversas fuentes documentales evidencian iniciativas de ornamentación de los espacios de la ciudad (e. g., PHILIPPI, 1882, 1884), así como la introducción de plantas exóticas para ornamentación de parques, plazas y calles (e. g., VICUÑA-MACKENNA, 1874). No obstante, este auge desde la partida no contempló el uso de los elementos de la flora nativa como sujetos de ornamentación, recurriéndose a especies principalmente asiáticas y europeas que terminaron por dominar estos espacios.

El interés declarado en el uso de especies de plantas nativas en la ornamentación de la ciudad es relativamente reciente, no tiene más de 30 años, y descansa sobre un reducido número de especies entre las que se cuentan principalmente a Acacia caven (espino), Cestrum parqui (parqui), Cryptocarya alba (peumo), Peumus boldus (boldo), Quillaja saponaria (quillay) y Schinus molle (pimiento). Importante es destacar el caso de la comuna de Santiago, pues representa el sector más antiguo de la ciudad. Los sitios muestreados al interior de esta comuna arrojaron bajos niveles de diversidad de especies de plantas. Aunque esta situación pudiese parecer contradictoria con el hecho de que la comuna de Santiago alberga grandes parques y paseos ornamentados, como el Cerro Santa Lucía, Parque Forestal y la Quinta Normal, es necesario destacar que la cartografía en base a IDW se basa en métodos que ponderan puntos vecinos; por tanto, la diversidad florística de estos parques y paseos es más bien restringida y no constituye una tendencia espacial de diversidad más allá de su ubicación.

Debido a que la representación de especies exóticas es mayor que la riqueza de especies nativas, la distribución espacial de las primeras se ajusta de mejor manera a la composición total de especies. Efectivamente, los sitios en que hubo mayor diversidad de especies exóticas también hubo mayor diversidad de especies totales (Figura 4). Estos focos de diversidad se correspondieron estrechamente con los focos de diversidad de especies totales. Las especies 
nativas, por su parte, ofrecen un menor número de especies ya que solo representan el $15 \%$ de la flora de Santiago. Cabe destacar, sin embargo, que la riqueza de especies nativas se encuentra más ampliamente distribuida al interior de Santiago, como lo muestra el número y extensión de áreas coloreadas en rojo (Figura 5).

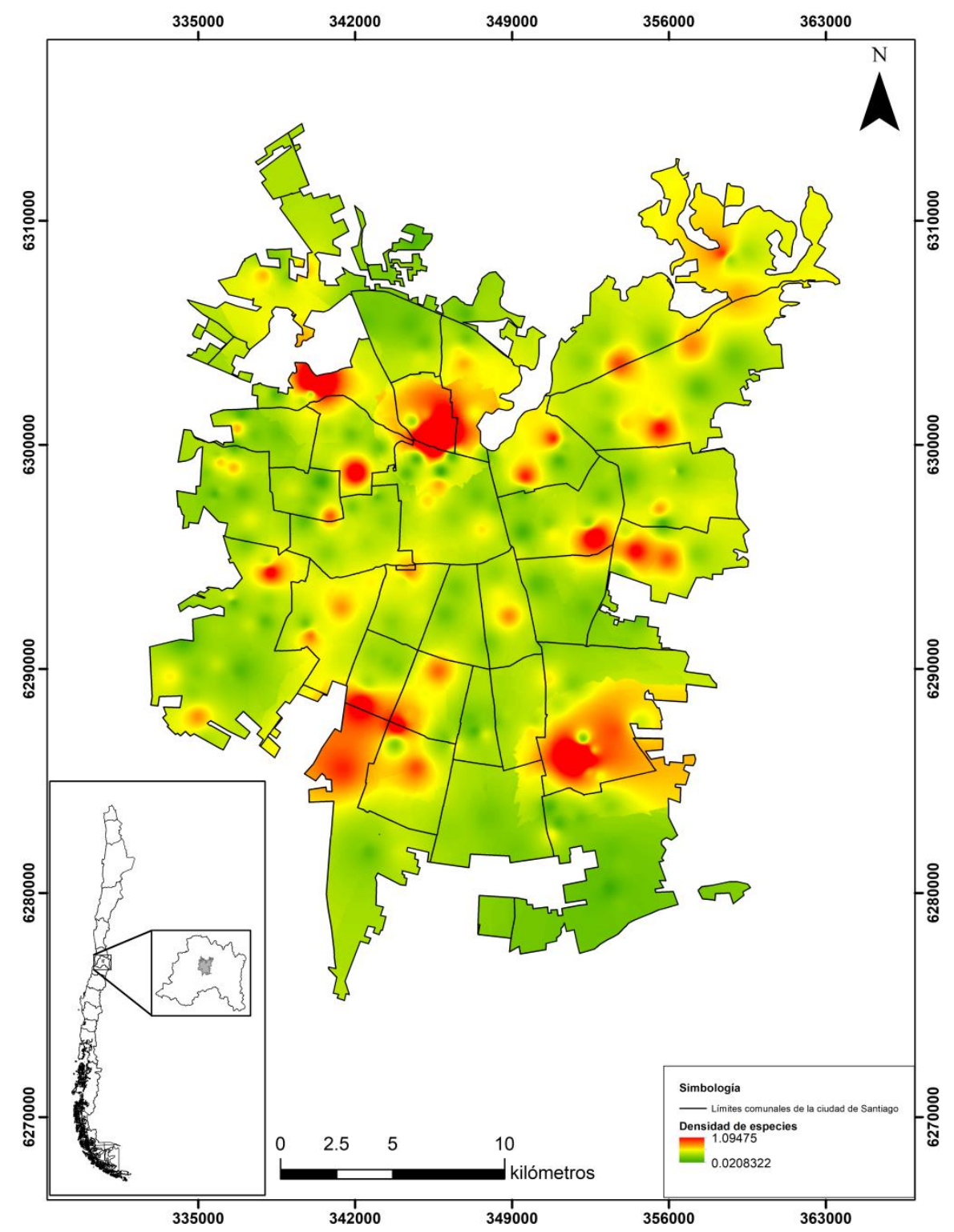

Figura 3. Interpolación IDW de la densidad de todas las especies de plantas al interior de la ciudad de Santiago.

La preponderancia de la riqueza de especies nativas y exóticas ha sido parcialmente analizada e informada en otras ciudades de Chile. En la mayor parte de los casos, tanto a nivel de cobertura espacial, así como de diversidad de especies la diversidad de especies exóticas superan a las nativas. Por ejemplo, Finot e Ramírez $(1996,1998)$ describieron la vegetación ruderal de la ciudad de Valdivia donde la representación de taxa exóticas alcanzó el 74 \% de la riqueza y las nativas el $26 \%$. Por su parte, San Martín et al. (2001) analizaron la composición de la flora asociada al estero Reñaca, documentando que el $64 \%$ de las especies fueron exóticas, las que alcanzaron el 76 \% de la cobertura vegetal; Rozzi et al. (2003) estudiaron 
cuatro plazas de Armas pertenecientes a cuatro ciudades del extremo sur de Chile, encontrando 503 árboles plantados como ornamentales, de los que el $99 \%$ pertenecieron a especies exóticas. En tanto que Pauchard et al. (2006) Ilamaron la atención acerca de la importante representación de especies exóticas en la ciudad de Concepción, un fenómeno que ya parecía ser recurrente en Chile.

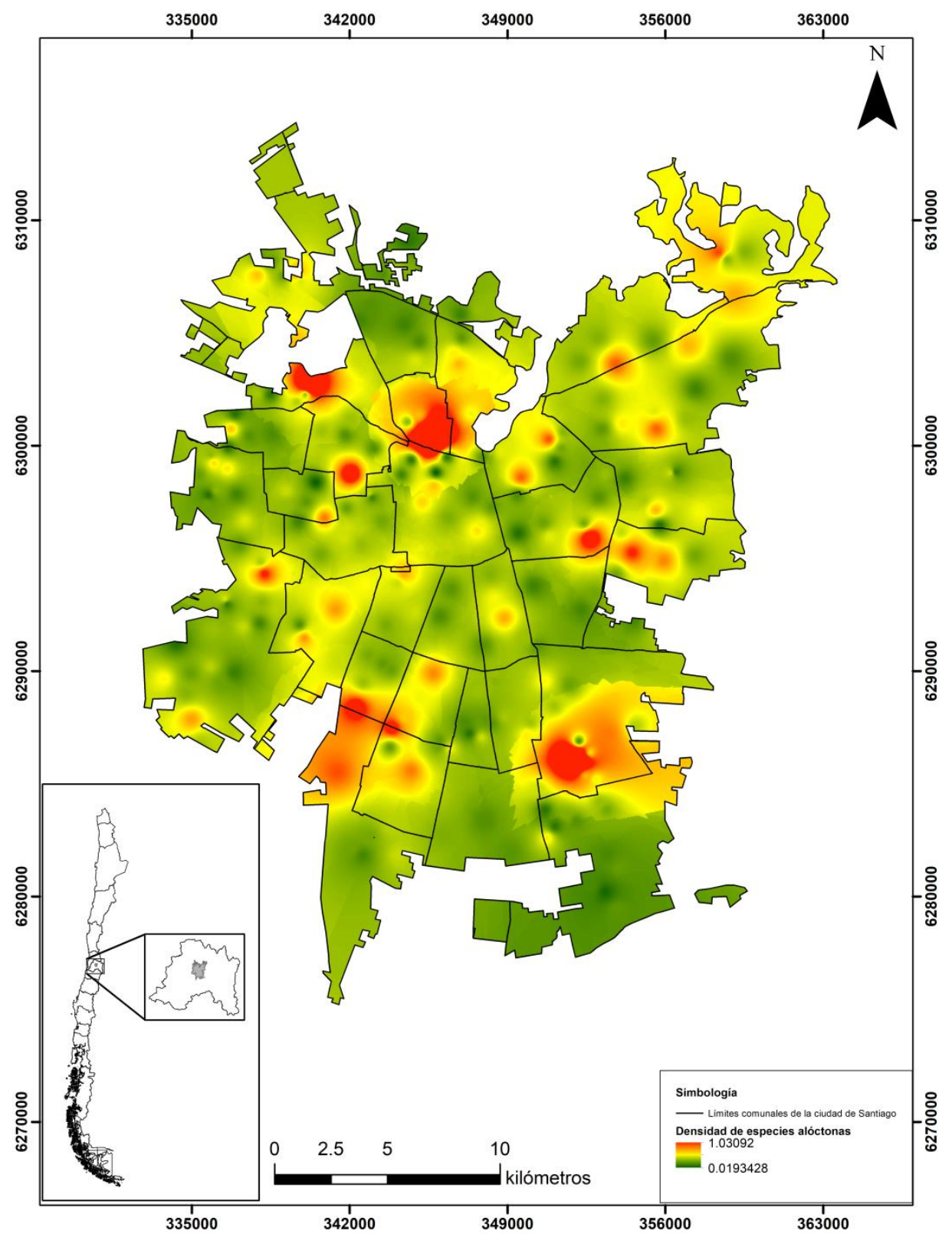

Figura 4. Interpolación IDW de la densidad de especies de plantas exóticas (también llamadas alóctonas) al interior de la ciudad de Santiago.

Más recientemente, Lozano-Diéguez e Teillier (2014) describieron la diversidad de especies leñosas en calles y plazas de Curicó, destacando que la representación de nativas y exóticas fue de $8 \%$ y $92 \%$, respectivamente. Fischer et al. (2016) analizando la composición de hierbas en prados de 15 parques de Santiago encontraron que la diversidad estuvo representada por un $90 \%$ de especies exóticas. A juzgar por la evidencia disponible para otras ciudades del globo (ver ARONSON et al., 2014, LA SORTE et al., 2014), la preponderancia de 
especies exóticas por sobre las nativas a nivel de la ciudad pero también a escala de los tipos de hábitat en su interior, constituye una situación inusual, pocas veces vista en otras urbes.

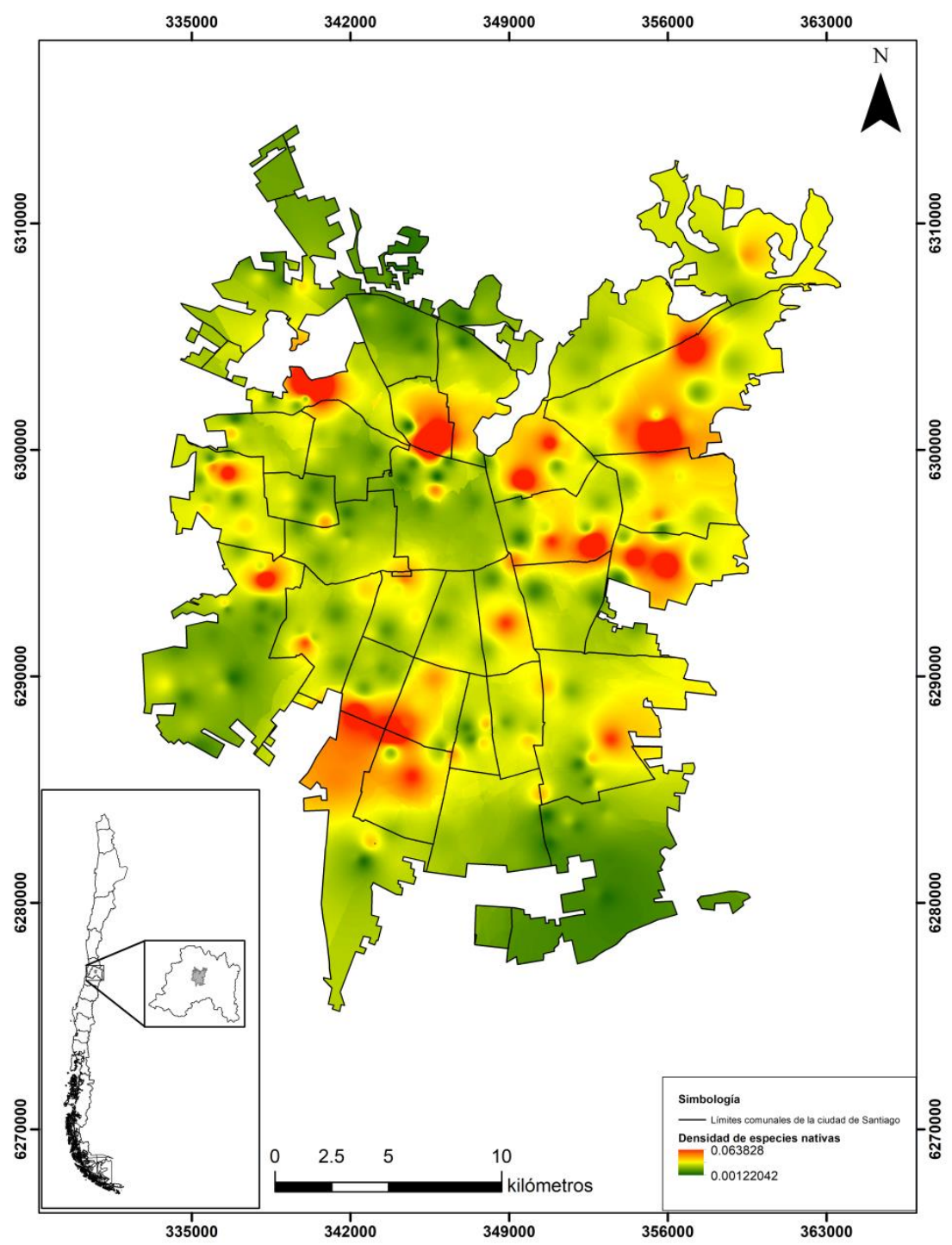

Figura 5. Interpolación IDW de la densidad de especies de plantas nativas al interior de la ciudad de Santiago.

Finalmente, es importante considerar que la representación de especies exóticas y nativas no es trivial para una ciudad como Santiago, pues esta urbe se emplaza en una región de importancia mundial en términos de diversidad florística (MYERS et al., 2000). En efecto, la región biogeográfica en la que se encuentra Chile central (30-36 S) contiene una flora nativa de carácter único por su alto nivel de endemismo (50 \%; ARROYO et al., 1999). No obstante, desde el punto de vista de la conservación de la flora se vislumbra un complejo panorama ya que el $70 \%$ de las ciudades del país albergando al $62 \%$ de la población (INE, 2005). Por tanto parece necesario implementar políticas que incentiven la plantación de especies nativas al interior de la ciudad con el objeto de establecer un mayor vínculo e integración con el medio geográfico. La flora de Santiago muestra una amplia representación de especies de plantas exóticas, en tanto que las especies nativas tienen una menor representación y extensión en su 
distribución espacial al interior de la ciudad. Este patrón composicional es concordante con lo documentado para otras ciudades del centro y sur de Chile, que son fuertemente dominadas por flora exótica. Por otra parte, nuestros resultados no concuerdan con ciudades del hemisferio norte, donde las especies nativas están representadas al menos por el $50 \%$ de las especies de plantas urbanas. Esta alta representación de especies exóticas en ciudades de Chile está determinada por factores de origen socio-cultural y ambiental como comprendidos.

\section{Agradecimientos}

Este estudio fue financiado por Programa DICYT 021543CM y Proyecto USA1555.48 de la Universidad de Santiago de Chile. Se agradece el apoyo del Centro para el Desarrollo de la Nanociencia y la Nanotecnología CEDENNA FB0807 (Línea 6), al Fondo Interno de Investigación CIP 16012, Universidad Central de Chile y al Programa Regular de Investigación CEAUP.

\section{Referencias}

1. ARAUJO, M.B. The coincidence of people and biodiversity in Europe. Global Ecology and Biogeography, v. 12, p. 5-12, 2003.

2. ARONSON, M.F. et al. A global analysis of the impacts of urbanization on bird and plant diversity reveals key anthropogenic drivers. Proceedings of the Royal Society B: Biological Sciences, 281, 20133330, 2014.

3. ARROYO, M.T.K. et al. Plant invasions in Chile: present patterns and future predictions. En: MOONEY, H.A.; HOBBS, R.J. (Eds). Invasive species in a changing world. Island Press, Covelo, California, p. 385-421, 2000.

4. ARROYO, M.T.K. et al. Central Chile. En: MITTERMEIER, R.A.; MYERS, N.; ROBLES-GIL, P.; GOETTSCH-MITTERMEIER, C. (Eds). Hotspots: Earth's biologically richest and most endangered terrestrial ecosystems. Cemex, Conservation International, Ciudad de Mexico, p. 184-199, 1999.

5. BROWN, D.G. et al. Rural land-use trends in the conterminous United States, 1950-2000. Ecological Applications, v. 15, p. 1851-1863, 2005.

6. CASTRO, S.A. et al. Minimum residence time, biogeographical origin, and life cycle as determinants of the geographical extent of naturalized plants in continental Chile. Diversity and Distributions, v. 11, p. 183-191, 2005.

7. CELESTI-GRAPOW, L. et al. The vascular flora of Rome. Plant Biosystems, v. 147, p. 10591087, 2013.

8. CINCOTTA, R.P.; WISNEWSKI, J.; ENGELMAN, R. Human population in the biodiversity hotspots. Nature, v. 404, p. 990-992, 2000.

9. COLLINS, J.P. et al. A new urban ecology. American Scientist, v. 88, p. 416-425, 2000.

10. CREUTZIGA, F. et al. Global typology of urban energy use and potentials for an urbanization mitigation wedge. Proceedings of the National Academy of Sciences, USA, v. 112, p. 6283-6288, 2015.

11. DE LA MAZA, C.L. et al. Vegetation diversity in the Santiago de Chile urban ecosystem. Arboricultural Journal, v. 26, p. 347-357, 2002.

12. ECKERT, S.; KOHLER, S. Urbanization and health in developing countries: a systematic review. World Health and Population, v. 15, p. 7-20, 2014. 
13. ELLIS, E.C. et al. Anthropogenic transformation of the biomes, 1700 to 2000 . Global Ecology and Biogeography, v. 19, p. 589-606, 2010.

14. ESCOBEDO, F. et al. The socioeconomics and management of Santiago de Chile's public urban forests. Urban Forestry and Urban Greening, v. 4, p. 105-114, 2006.

15. FELSON, A.J. et al. Mapping the design process for urban ecology researchers. BioScience, v. 63, p. 854-865, 2013.

16. FIGUEROA, J.A. et al. Vascular flora in public space of Santiago, Chile. Gayana Botánica (Chile), v. 73, p. 85-103, 2014.

17. FINOT, V.L.; RAMíREZ, C. Fitosociología de la vegetación ruderal de la ciudad de Valdivia (X Región, Chile) 1. Vegetación nitrófila. Stvdia Botanica (Spain), v. 15, p. 159-170, 1996.

18. FINOT, V.L.; RAMíREZ, C. 1998. Fitosociología de la vegetación ruderal de la ciudad de Valdivia (X Región, Chile) 2. Vegetación de senderos. Stvdia Botanica (Spain), v. 17, p. 6986, 1998.

19. FISCHER, L.K. et al. Drivers of biodiversity patterns in parks of a growing South American megacity. Urban Ecosystem, v. 19, p. 1231-1249, 2016.

20. GARTNER, E.; ROJAS, G.; CASTRO, S. A. Compositional patterns of ruderal herbs in Santiago, Chile. Gayana Botánica (Chile), v. 72, p. 192-202, 2015.

21. GASTON, K.J. et al. Urban domestic gardens (IV): the extent of the resource and its associated features. Biodiversity and Conservation, v. 14, p. 3327-3349, 2005.

22. GRIMM, N.B. et al. Global change and the ecology of cities. Science, v. 319, p. 756-760, p. 2008.

23. HERNÁNDEZ, H.J.; VILLASEÑOR, N.R. Twelve-year change in tree diversity and spatial segregation in the Mediterranean city of Santiago, Chile. Urban Forestry \& Urban Greening, v. 29, p. 10-18, 2017.

24. HOFFMANN, A. El árbol urbano en Chile. 3a edición. Fundación Claudio Gay. Santiago, Chile. 255 p. 1998.

25. INE. Instituto Nacional de Estadísticas. Chile: ciudades, pueblos, aldeas y caseríos. Departamento de Geografía y Censos. Santiago, Chile. 300 p. 2005.

26. KABISCH, N.; HAASE, D. Diversifying European agglomerations: evidence of urban population trends for the 21st century. Population, Space and Place, v. 17, p. 236-253, 2011.

27. KNAPP, S. et al. Challenging urban species diversity: contrasting phyllogenetic patterns across plant functional groups in Germany. Ecology Letters, v. 11, p. 1054-1064, 2008.

28. KOWARIK, I.; VON DER LIPPE, M.; CIERJACKS, A. Prevalence of alien versus native species of woody plants in Berlin differs between habitats and at different scales. Preslia, v. 85, p. 113-132, 2013.

29. KOWARIK, I. Novel urban ecosystems, biodiversity, and conservation. Environmental Pollution, v. 159, p. 1974-1983, 2011.

30. KÜHN, I.; BRANDL, R.; KLOTZ, S. The flora of German cities is naturally species rich. Evolutionary Ecology Research, v. 6, p. 749-764, 2004.

31. LA SORTE, F.A. et al. Beta diversity of urban floras among European and non-European cities. Global Ecology and Biogeography, v. 17, p. 363-371, 2014.

32. LA SORTE, F.; PYSEK, P. Extra-regional residence time as a correlate of plant invasiveness: European archaeophytes in the North American flora. Ecology, v. 90, p. 2589-2597, 2009. 
33. LOSOSOVÁ, Z. et al. Diversity of Central European urban biota: effects of human-made habitat types on plants and land snails. Journal of Biogeography, v. 38, p. 1152-1163, 2011.

34. LOZANO-DIÉGUEZ, A.; TEILLIER, S. Frecuencia y abundancia de especies leñosas utilizadas en espacios públicos de la ciudad de Curicó, Región del Maule, Chile. Chloris Chilensis, v. 17, 2014.

35. MACGREGOR-FORS, I.; ORTEGA-ALVAREZ, R. Ecología urbana: experiencias en América Latina. Disponible en línea: www1.inecol.edu.mx/libro_ecologia_urbana (ISBN: 978-60700-6869-0). 130 p. 2013.

36. MARCO, A. et al. Bridging human and natural sciences for a better understanding of urban floral patterns: The role of planting practices in Mediterranean gardens. Ecology and Society, v. 15, p. 2, 2010.

37. MARTICORENA, C.; QUEZADA, M. Catálogo de la flora vascular de Chile. Gayana Botánica (Chile), v. 42, p. 5-157, 1985.

38. MARTICORENA, C.; QUEZADA, M. Adiciones a la flora de Chile. Gayana Botánica (Chile), v. 44, p. 39-44, 1987.

39. MATTHEI, O. Manual de las malezas que crecen en Chile. Alfabeta Impresores, Santiago, Chile. 545 p. 1995.

40. MCDONALD, R. I.; MARCOTULLIO, P. J. Urbanization and Global Trends in Biodiversity and Ecosystem Services. En: ELMQVIST, T. et al. (Eds). Urbanization, biodiversity and ecosystem services: challenges and opportunities: a global assessment. Springer Open, p. 31-52, 2013.

41. MCDONALD, R. I. Global urbanization: can ecologists identify a sustainable way forward? Frontiers in Ecology and the Environment, v. 6, p. 99-104, 2008.

42. MCDONNELL, M.J.; HAHS, A.K. 2009. Comparative ecology of cities and towns: past, present and future. En: MCDONNELL, M.J; HAHS, A.K; BREUSTE, J. (Eds). Ecology of cities and towns: a comparative approach. Cambridge University Press, Cambridge, UK. p. 7189, 2009.

43. MCKINNEY, M.L. Urbanization, biodiversity, and conservation. Bioscience, v. 52, p. 883890, 2002.

44. MCKINNEY, M.L. Effects of urbanization on species richness: a review of plants and animals. Urban Ecosystems, v. 11, p. 161-176, 2008.

45. MYERS, N. et al. Biodiversity hotspots for conservation priorities. Nature, v. 40, 853-858, 2000.

46. PAUCHARD, A. et al. Multiple effects of urbanization on the biodiversity of developing countries: the case of a fast-growing metropolitan area (Concepción, Chile). Biological Conservation, v. 127, p. 272-281, 2006.

47. PHILIPPI, F. Memoria y catálogo de las plantas cultivadas en el jardín Botánico hasta el 1o de mayo de 1884. Imprenta Nacimiento. Santiago. 83 p. 1884.

48. PHILIPPI, R.A. Catálogo de las plantas cultivadas para el Jardín Botánico de Santiago hasta el 1 ㅇ de mayo de 1881. Imprenta Nacimiento. Santiago. 55 p. 1882.

49. PICKETT, S.T.A. et al. Urban ecological systems: scientific foundations and a decade of progress. Journal of Environmental Management, v. 92, p. 331-362, 2011. 
50. PICKETT, S.T.A. et al. Urban ecological systems: linking terrestrial ecological, physical, and socioeconomic components of metropolitan areas. Annual Review of Ecology and Systematics, v. 32, p. 127-157, 2001.

51. POUYAT, R.V. et al. Urban land-use change effects on biogeochemical cycles. En: CANADELL, P.; PATAKI, D.E.; PITELKA, L. (Eds). Terrestrial ecosystems in a changing world. Springer-Verlag Berlin, Germany. p. 45-58, 2007.

52. RAY, C. et al. Catálogo de la flora y fauna invasora en Chile continental e insular. En: JAKSIC, F.M.; CASTRO, S.A. (Eds). Invasiones Biológicas en Chile: causas globales e impactos locales. Pontificia Universidad Católica de Chile, Santiago (Chile). p. 491-509, 2014.

53. REDMAN, C.L.; GROVE, J.M.; KUBY, L.H. Integrating social science into the long-term ecological research (LTER) network: social dimensions of ecological change and ecological dimensions of social change. Ecosystems, v. 7, p. 161-171, 2004.

54. REYES-PAECKE, S.; MEZA, L. Residential gardens of Santiago, Chile: extent, distribution and vegetation cover. Revista Chilena de Historia Natural, v. 84, p. 581-592, 2011.

55. RODRÍGUEZ, R.; RUIZ, E.; ELISSETCHE, J.P. Arboles en Chile. Universidad de Concepción. Concepción, Chile. 183 p. 2005.

56. ROZZI, R. et al. Árboles nativos y exóticos en las plazas de Magallanes. Anales Instituto Patagonia (Chile), v. 31, p. 27-42, 2003.

57. SAN MARTín, C. et al. Flora y vegetación del estero Reñaca (V Region, Chile). Gayana Botánica (Chile), v. 58, p. 31-46, 2001.

58. SCHNEIDER, A.; FRIEDL, M.A.; POTERE, D. Mapping global urban areas using MODIS 500$\mathrm{m}$ data: new methods and databases. Remote Sensing of Environment, v. 114, p. 17331746, 2010.

59. SHOCHAT, E. et al. Invasion, competition, and biodiversity loss in urban ecosystems. BioScience, v. 60, p. 199-208, 2010.

60. TURNER, W.R.; NAKAMURA, T.; DINETTI, M. Global urbanization and the separation of humans from nature. BioScience, v. 54, p. 585-590, 2004.

61. UN. United Nations. World urbanization prospects: the 2007 Revision. New York. 244 p. 2007.

62. UN. United Nations. World population prospects: the 2010 Revision. New York. $481 \mathrm{p}$. 2011.

63. VICUÑA-MACKENNA, B. Álbum del Santa Lucía: colección de las principales vistas monumentos, jardines estatuas y obras de arte de este paseo: dedicado a la Municipalidad de Santiago por su actual presidente B. Vicuña Mackenna. Santiago de Chile. Imprenta de la Librería del Mercurio. 46 p. 1874.

64. WATSON, D.F.; PHILIP, G.M. A refinement of inverse distance weighted interpolation. Geoprocessing, v. 2, p. 315-327, 1985.

65. WERNER, P. The ecology of urban areas and their functions for species diversity. Landscape and Ecological Engineering, v. 7, p. 231-240, 2011. 